\title{
Infective endocarditis after tattooing in adolescent patient with ventricular septal defect
}

\author{
Çetin Alak ${ }^{1}$
}

1) Dokuz Eylül University Faculty of Medicine, Department of Cardiology, Dr., Izmir, Turkey

\begin{abstract}
Congenital heart disease (CHD), valvular diseases and medical procedures are some of well-known predisposing factors for infective endocarditis (IE). IE after tattooing is uncommon complication comparing local infections, allergic reactions etc. But, advances made in diagnosis and treatment of CHD result an increasing adult CHD population and tattooing is also becoming more popular in adolescent and young adults. Therefore, IE after tattooing is likely to be seen more. Herein, we report a patient with ventricular septal defect who developed IE after tattooing.
\end{abstract}

Keywords: Infective endocarditis, tattooing, congenital heart disease, ventricular septal defect 


\section{Introduction}

Infective endocarditis (IE) is a dangerous complication of body arts in patients with and without congenital heart disease (CHD). ${ }^{1}$ Tattooing and body piercing has been becoming more popular and socially acceptable. Therefore, IE cases associated with body art continue to be reported in the literature. According to our knowledge, only three cases with CHD and IE after tattooing have been reported to date. ${ }^{2-4}$ Herein, we report a patient with ventricular septal defect (VSD) who developed IE after tattooing.

Figure 1. Image of the patient's right arm with tattoo.

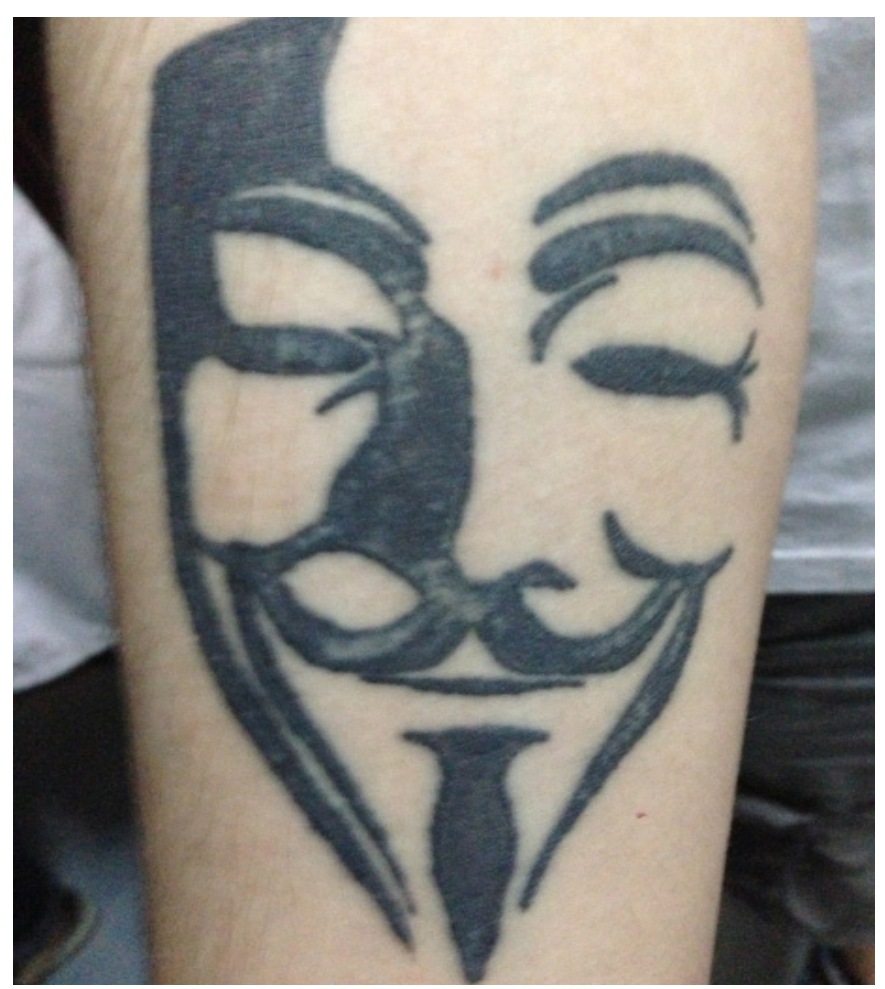

Figure 2a. Transesophageal echocardiography demon strating mobile mass $(18 \times 6 \mathrm{~mm})$ attached to right ventricular outflow track.

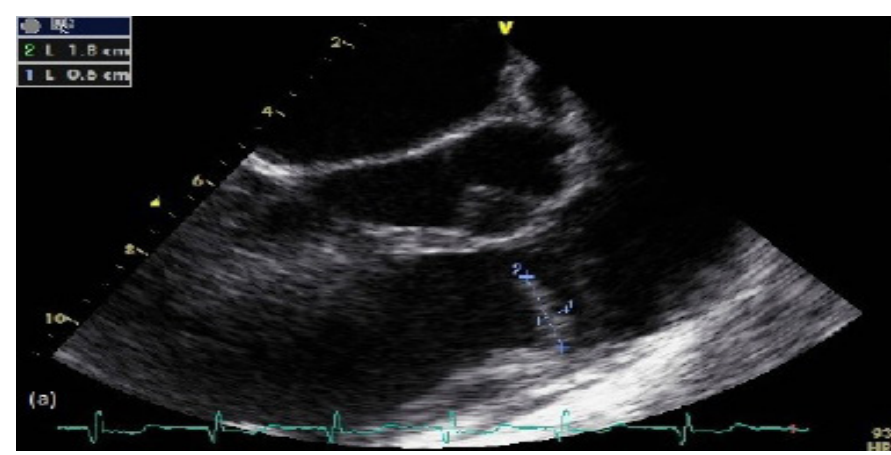

\section{Case presentation}

A nineteen-year-old male patient with no prior medical history started having widespread rash a week after getting tattoo to his right arm (Figure 1). He was admitted to dermatology department. Although rashes tended to disappear with prednisolone, nausea, vomiting and fever episodes began. He was referred to our clinic due to hearing of serious cardiac murmur. In his detailed medical history, he denied erythema, swelling, and discharge after tattooing and intravenous drug abuse.

Physical examination revealed body temperature of $39.5^{\circ} \mathrm{C}$, heart rate of 120 beats $/ \mathrm{min}$, blood pressure of 105/65 mmHg and IV/VI pansystolic murmur ant thrill on mesocardiac region. On laboratory tests, hemoglobin level was $9.11 \mathrm{~g} / \mathrm{dl}$, white blood cell count 21,700 (K/ $\mu \mathrm{l})$, platelet count $305,000(\mathrm{~K} / \mu \mathrm{l})$, serum urea $28 \mathrm{mg} /$ $\mathrm{dl}$, and serum creatinine $0.93 \mathrm{mg} / \mathrm{dl}$. Sedimentation rate was $89 \mathrm{~mm} / \mathrm{h}$ and CRP was $19 \mathrm{mg} / \mathrm{dl}$ (Normal range: $0-0.3 \mathrm{mg} / \mathrm{dl})$.

Transthoracic and transesophageal echocardiography was performed revealing mobile mass $(18 \times 6 \mathrm{~mm})$ attached to right ventricular outflow track consistent with endocarditis (Figure 2a) and $10-\mathrm{mm}$ ventricular septal defect with left to right shunt (Figure 2b). Qp/ Qs was calculated as 1.6. Five blood cultures were collected from the patient; three samples on the first and two samples on the second day of administration to hospital. He was empirically started on cefazolin.

Blood was cultured in BACTEC (Beckton Dickinson, USA) and incubated in BACTEC FX 200 (Beckton Dickinson, USA). Identification and antibiotic sus-

\section{Figure 2b. Color Doppler image showing 10-mm ventricular septal defect causing turbulent flow.}

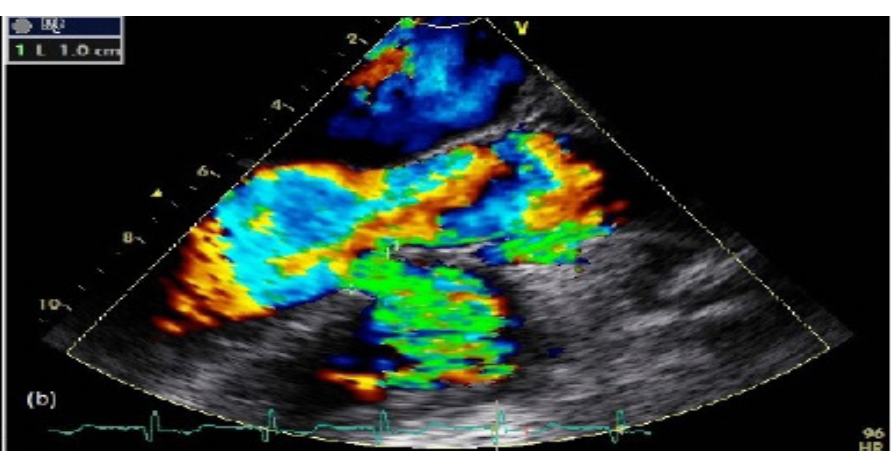


ceptibilities were done by BD Phoenix 100 (Beckton Dickinson, USA). All blood cultures were positive for Streptococcus sangius. Daptomycin was substituted for cefazolin. The operation was performed at non-active period after antibiotherapy. The defect was directly closed and the vegetation was excised totally. After 6-week treatment, he was discharged with no complaint.

\section{Discussion}

Infective endocarditis is still related with high mortality, despite advances in diagnostic methods, antibiotic treatment, and surgical treatment options. Lots of causative microorganisms were reported. Valvular and congenital heart diseases (CHD) are common predisposing factors for IE. ${ }^{5-7}$ Advances made in diagnosis and treatment of CHD result an increasing adult CHD population. Transient bacteriemia occurring after skin break can lead to IE particularly in individuals with
CHD. Tattooing is a fashionable practice involving skin breaks up to 150 times a second. It can result superficial and deep local infections, systemic infections, allergic reactions, granulomatous reactions and other skin diseases such as eczema, psoriasis etc. ${ }^{8}$

Although IE following tattooing remains an uncommon complication, its incidence is likely to be raised with increasing adult CHD population and popularity of tattooing. Therefore education of patients and body art professionals about good hygienic practice and wound care is vital, especially in patients with CHD.

In conclusion, non-medical procedures; such as tattooing, piercing can be a risk factor for development of IE especially in patients with structural cardiac abnormalities. Consequently, more attention should be paid to aseptic techniques during the procedures in patients with CHD. 


\section{References}

1. Armstrong ML, DeBoer S, Cetta F. Infective endocarditis after body art: a review of the literature and concerns. The Journal of adolescent health : official publication of the Society for Adolescent Medicine. Sep 2008;43(3):217-225.

2. Satchithananda DK, Walsh J, Schofield PM. Bacterial endocarditis following repeated tattooing. Heart. Jan 2001;85(1):11-12.

3. Tse D, Khan S, Clarke S. Bacterial endocarditis complicating body art. International journal of cardiology. Mar 20 2009;133(1):e28-29.

4. Akkus NI, Mina GS, Fereidoon S, et al. Tattooing complicated by multivalvular bacterial endocarditis. Herz. May 2014;39(3):349-351.

5. McKinsey DS, Ratts TE, Bisno AL. Underlying cardiac lesions in adults with infective endocarditis. The changing spectrum. The American journal of medicine. Apr 1987;82(4):681-688.

6. Gersony WM, Hayes CJ, Driscoll DJ, et al. Bacterial endocarditis in patients with aortic stenosis, pulmonary stenosis, or ventricular septal defect. Circulation. Feb 1993;87(2 Suppl):I121-126.

7. Agca N, Peker T, Ari H, et al. Infective endocarditis: a tertiary referral centre experience from Turkey. International journal of clinical and experimental medicine. 2015;8(8):13962-13968.

8. Khunger N, Molpariya A, Khunger A. Complications of Tattoos and Tattoo Removal: Stop and Think Before you ink. Journal of cutaneous and aesthetic surgery. Jan-Mar 2015;8(1):30-36.

Received: $21 / 03 / 2016$

Accepted: 14/08/2016

Published: 15.09 .2016

Disclosure and conflicts of interest:

Conflicts of interest were not reported.

\section{Corresponding author:}

Dr. Çetin Alak

Mail: cetinalak@mynet.com 